\title{
Formulación de modelos de gestión del conocimiento aplicados al contexto de instituciones de educación superior
}

\author{
Yesenia Acevedo-Correa ${ }^{1 \star}$, Carlos A. Aristizábal-Botero ${ }^{2}$, Alejandro Valencia-Arias ${ }^{1}$, Lemy Bran-Piedrahita1 \\ (1) Coordinación General de Investigaciones, Fundación Universitaria Católica del Norte. Avenida 33 \# 74 E 51 (Centro \\ de Servicio Educativo) Medellín - Colombia. (e-mail: yacevedoc@ucn.edu.co; Ibpiedrahita@ucn.edu.co; \\ javalenciaa@ucn.edu.co) \\ (2) Departamento de Sociología, Universidad de Antioquia. Calle 70 No. 52 - 21. Bloque 9-228 Medellín - Colombia. (e- \\ mail: carlos.aristizabal@udea.edu.co)
}

* Autor a quien debe ser dirigida la correspondencia.

Recibido Jun. 5, 2019; Aceptado Jul. 31, 2019; Versión final Sep. 30, 2019, Publicado Feb. 2020

\section{Resumen}

El propósito de este artículo es plantear una propuesta metodológica para la formulación de modelos de gestión del conocimiento aplicados al contexto de Instituciones de Educación Superior. Esta propuesta se validó con información de la Fundación Universitaria Católica del Norte, de Medellín-Colombia. La metodología es una ruta constituida por: diagnóstico, diseño, implementación y validación de estrategias. Se validaron teóricamente las dimensiones y categorías del modelo, así como su aplicabilidad a través de talleres interactivos, bajo el método del diseño de pensamiento. Entre los principales resultados se observa la utilidad de realizar un diagnóstico previo con el fin de contextualizar el panorama de intervención, y generar mapas de conocimiento orientados a promover la concientización de los procedimientos institucionales como mecanismo para explicitar y apropiar los conocimientos relevantes.

\section{Formulation of knowledge management models applied to the context of higher education institutions}

\begin{abstract}
The purpose of this article is to analyze a methodological proposal for the formulation of knowledge management models applied to the context of Higher Education Institutions. The proposal was validated in the Fundación Universitaria Católica del Norte, de Medellin-Colombia. The methodology is a path consisting of diagnosis, design, implementation and validation of strategies. The dimensions and categories of the model were theoretically validated, as well as their applicability through interactive workshops, under the method of thought design. Among the main results is the usefulness of making a previous diagnosis in order to contextualize the intervention landscape and generate knowledge maps aimed at raising awareness of institutional procedures such as mechanism to explain and appropriate relevant knowledge.
\end{abstract}

Keywords: higher education; resources management; knowledge management; know how transfer 


\section{INTRODUCCIÓN}

Para acercarse a la gestión del conocimiento (GC) en Instituciones de Educación Superior (IES), es indispensable comenzar mostrando algunos de sus enfoques en el contexto universitario. Al respecto, se identifican diferentes posturas de GC, una de estas se encuentra en la revisión teórica que realizan los autores Arévalo-Avecillas, et al. (2018), quienes mencionan que la GC es un componente principal para las empresas de servicios, en las cuales la GC determina las destrezas para brindar nuevos servicios y generar reconocimiento en el mercado, para ello es crucial la inversión en investigación, que sirve para medir niveles de productividad en las organizaciones y así ser competitivas a largo y mediano plazo. Por otra parte, el estudio realizado por Marulanda, et al. (2019), reconocen la GC como de los activos más importantes para cualquier institución empresarial o académica, este se comprende como el proceso para almacenar, capturar, compartir y utilizar el conocimiento para lograr ventajas competitivas. Lo anterior se logra a partir del desarrollo de una cultura intensiva en conocimiento, por lo tanto, debe buscarse la promoción de procesos que faciliten la interacción e intercambio de conocimientos (Marulanda, et al. 2019). De Molero, et al. (2017), plantean que la investigación puede aumentar su productividad a través de la gestión del conocimiento con espacios de interacción, discusión, renovación y actualización, pues se señalan necesidades a resolver para mejorar el entorno social, y para ello, las TIC son herramientas para diversificar los conocimientos y lograr su captura y transferencia.

Las instituciones de Educación Superior (IES) son señaladas por la Organización de las Naciones Unidas para la Educación, la Ciencia y la Cultura -UNESCO- (2009) como dinamizadoras de las capacidades sociales, económicas y personales, tanto de sus integrantes como de la sociedad. Por sus condiciones las IES posibilitan la transmisión, distribución y generación de conocimiento, recurso indispensable para participar de la resolución de los retos globales: seguridad alimentaria, cambio climático, gestión del agua, entre otros. Dichos procesos deben ser gestionados y ordenados de forma idónea con el fin de que se produzcan los resultados esperados. En este sentido, las IES en conjunto con otros actores contribuyen al desarrollo innovador de un país, siendo motor de cambio (De Moortel y Crispeels, 2018), además son fundamentales para la circulación y diseminación del conocimiento, pues como lo reseñan Rodríguez, et al. (2014) tienen la tarea de formar al capital humano encargado de desarrollar e innovar en las soluciones del planeta, es por ello, necesario que en las IES se diseñen estrategias de gestión, las cuales permitan incorporar acciones que posibiliten que el conocimiento que se genera en ellas, sea este científico, organizacional, tecnológico, o de otra índole, pueda redundar en el desarrollo de capacidades y aprendizajes para la institución.

González, et al. (2004) consideran que la gestión tradicional educativa que ha caracterizado a muchas IES se ha orientado a administrar el cumplimiento de condiciones curriculares reglamentadas por el Estado, más que a gestionar y sistematizar los aprendizajes y conocimientos generados, de tal forma que pueda ser transferido a la sociedad. Es por eso que explorar alternativas para aportar a la gestión, permite promover el desarrollo de estrategias para reconocer y acreditar los conocimientos y aprendizajes que se crean en los diferentes procesos universitarios, con la intención de que estos puedan ser usados estratégicamente (Asma y Abdellatif, 2016). Para ello, es necesario tener en cuenta que el conocimiento es considerado un bien intangible en la sociedad actual, que opera como herramienta competitiva, por lo cual, precisa ser gestionado adecuadamente (Michelino, et al., 2015). En este caso, la gestión del conocimiento se asume como la manera en la que se ponen a disposición, de manera ordenada, práctica y eficaz, de las instituciones el conocimiento tácito (empírico) y explícito (formalizado), con la intención de mejorar su productividad y funcionamiento (De Molero, et al., 2017) y, para alcanzar tal objetivo, es necesario hacer uso de recursos metodológicos de la gestión de conocimiento (en adelante GC), pues con ellos es posible no solo reconocer y acreditar aprendizajes y conocimientos, sino también crear valor. Para ello, deben diseñarse estrategias para implementar eficientemente el modelo de GC, pues su estrategia contribuye a al proceso de creación, documentación y comunicación de información tácita y explícita en la organización (Hosseini, et al., 2019) que permitan: crear, codificar, apropiar, compartir y reutilizar el conocimiento (Nonaka y Takeuchi, 1999) de tal manera que posibiliten el desarrollo de trabajo colectivo, y que aporte a la adquisición de habilidades en todos los niveles de la organización (Yusof, et al., 2012). Así las cosas, para hablar de un modelo de gestión del conocimiento, se hace referencia al diseño e implementación de acciones en los diferentes procesos organizacionales, además, al sistema de trabajo que apoye y promueva el almacenamiento, la transferencia y la aplicación del conocimiento (Villafuerte-Godínez y Leiva, 2015).

La gestión del conocimiento en IES tiene que ver con direccionar sus activos cognitivos, como bienes estratégicos que generan oportunidades para el desarrollo de las actividades sustantivas -docencia, investigación, extensión y proyección social-, así como para generar y mantener la calidad institucional, pues este permea sus procesos (Rodríguez, et al., 2014). Por tanto, la GC vincula personas y tecnologías que promueven políticas y prácticas para compartir y apropiar el conocimiento (Rodríguez, et al., 2014). De acuerdo a lo anterior, las IES han implementado modelos de gestión del conocimiento (Sein, Fidalgo, et al., 2017; Natek y Zwilling, 2014; Asma, y Abdellatif, 2016); los cuales parten del estudio de los contextos, en los que los individuos desarrollan su labor al interior de la IES, ello con el fin de implementar acciones que estimulen entornos para generar, aprender y compartir conocimiento (Moffet, et al., 2002; Chen y Burnstein, 
2006; Saad, et al., 2012) y se fortalezcan las funciones de docencia, extensión e investigación. Estos modelos implementan mecanismos para capturar datos e información de la institución, que a su vez puedan ser compartidos entre individuos, grupos y departamentos, los cuales esperan desarrollar cultura de gestión de conocimiento (Simanjuntak, et al., 2016) y es precisamente desde estos principios que se propondrán estrategias.

Al consultar los antecedentes institucionales de la Fundación Universitaria Católica del Norte (en adelante FUCN), respecto a acciones relacionadas con la GC se encontraron dos documentos; el primero presenta los resultados de valoración de las condiciones individuales, que podrían posibilitar compartir conocimiento al interior de la universidad (Roldán y Hernández, 2011), el segundo, presenta el diagnóstico sobre las dificultades institucionales para almacenar y conservar la información (Sánchez, 2016). Sin embargo, pese a la existencia de estos textos, no se avanzó en proponer estrategias de GC, puesto que el interés estuvo en valorar las condiciones institucionales. Estos trabajos demuestran el interés que ha tenido la FUCN por estudiar las condiciones que hacen posible la consolidación de un modelo de gestión de conocimiento, que permita dinamizar los aprendizajes y conocimientos que en ella se crean, pero además en aportar al desarrollo de estrategias que puedan ser implementadas en las universidades.

Se entiende aquí, que, en el caso de la FUCN, la cual desempeña su labor en ambientes virtuales, requiere del diseño de un Modelo Institucional de GC, que, en medio de un mundo globalizado y conectado, de grandes transformaciones y cambios permanentes, permita reconocer su compromiso con ser una institución de conocimiento cada vez más sólida (FUCN, 2016). Por ello, una forma de hacerlo es por medio de la implementación de estrategias de GC, las cuales han sido inspiradas en las teorías de Nonaka y Takeuchi (1999). Estos autores proponen un modelo que es presentado en la figura 1, compuesto por dos dimensiones para la creación de conocimiento, la primera denominada ontológica, que se refiere a los niveles tanto individual como colectivo; a esta pertenecen los diferentes tipos de relacionamiento (individual, grupal, organizacional e interorganizacional) que se dan al interior de la IES. La segunda dimensión denominada epistemológica, se relaciona con el tipo de conocimiento que los individuos poseen: tácito (de carácter individual y no externalizado) y explícito (externalizado y formalizado). Ambas dimensiones interactúan constantemente, por medio de la activación de los siguientes procesos: socialización (compartir conocimiento tácito entre pares), exteriorización (explicitar el conocimiento, por medio de su codificación), combinación (conjugar conocimientos explícitos) y la interiorización (la incorporación del conocimiento como un nuevo conocimiento tácito). A partir de la puesta en marcha de los diferentes procesos, se moviliza tanto la generación como la gestión de conocimiento, que finalmente, repercute de manera positiva en el total de la institución.

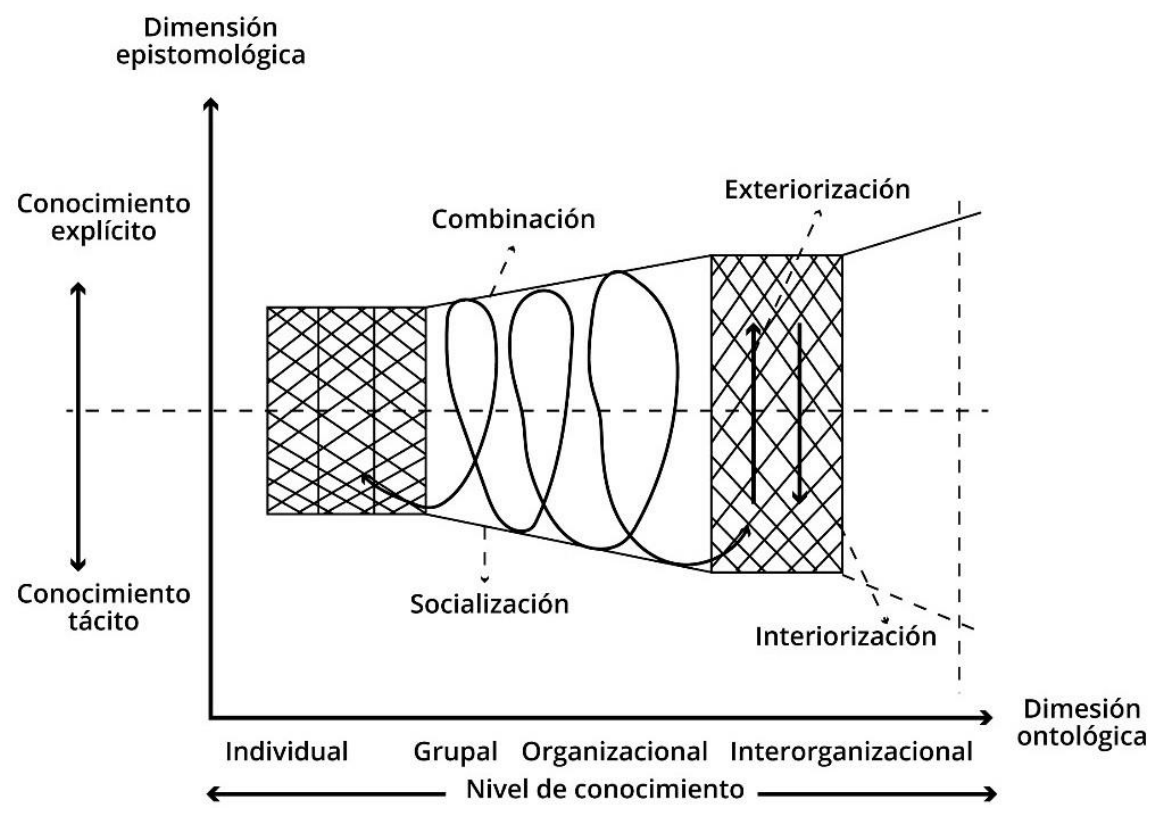

Fig. 1: Espiral de creación del conocimiento organizacional (Tomado de Nonaka y Takeuchi, 1999).

De acuerdo a lo anterior, y para dar cuenta del propósito de esta comunicación, el articulo presenta el desarrollo metodológico realizado en el contexto especifico de la FUCN, posteriormente se encuentran los resultados obtenidos en la fase de diagnóstico, organizados de acuerdo a tres capacidades identificadas para la institución universitaria: tecnológica, humana y organizacional, determinando en ellas los procesos organizacionales que podían ser intervenidos con estrategias de GC, luego se exponen las estrategias implementadas y finalmente se presentan las conclusiones entre las que se resalta la importancia de generar diagnósticos para la aplicación de estrategias de acuerdo al contexto de interés. 


\section{METODOLOGÍA}

La metodología constó con un enfoque cuantitativo, de corte transversal para analizar el caso específico y se centró en la construcción de una ruta constituida por tres fases (ver Figura 2): diagnóstico, diseño, implementación y validación de estrategias (inspiradas en la revisión de otras aplicaciones de modelos de GC). Inicialmente se tuvo como objetivo identificar actividades institucionales en las cuales era posible realizar intervenciones que promovieran la GC al interior de la institución. Para ello se revisó información documental institucional y, al mismo, tiempo se valoró el mapa de procesos organizacionales de la universidad, con el fin de determinar la manera en que podrían incorporarse estrategias. Además se realizó una revisión sistemática de cómo se promueve la gestión del conocimiento en contextos universitarios, y por último se recolectó información empírica por medio de una encuesta de percepción tipo Likert (Nunca; A veces; Generalmente; Muchas veces; Siempre) validada con una prueba piloto para ajustes y luego por dos expertos metodológicos de la Universidad de Antioquia para identificar capacidades organizacionales como tecnológica, humana y organizacional (Arias y Aristizábal, 2011) que se encuentra inspirada en los criterios propuestos por Nonaka y Takeuchi (1999). Con esto se revisaron componentes de condiciones de la GC en la organización. Así, los datos recolectados con este instrumento permitieron por una parte tener una visión general del funcionamiento organizacional y por otra, las condiciones en las cuales se encontraban la: exteriorización, socialización, combinación e interiorización del conocimiento. Esta prueba se aplicó a una muestra de 62 personas (directivos, coordinadores, administrativos y docentes) de un universo de 200 empleados con contrato laboral, para un valor de confianza del $1.28(90 \%)$ y se tuvo consentimientos informados en los que se manifestó que el estudio tenía fines netamente académicos. Para el análisis de los datos, se cotejaron percepciones de los encuestados con lo que se presenta teóricamente para determinar vacíos en la GC dentro de la estructura organizacional.

Producto del diagnóstico se diseñaron tres estrategias de GC, orientadas en el modelo SECI (Nonaka y Takeuchi, 1999). La elección de este como referente, radica en que, por un lado, incorpora una dimensión ontológica como parte de procesos de gestión, y por otro propone el aprendizaje como eje del proceso de gestión. Las estrategias propuestas fueron: mapas de conocimiento cuyo fin fue explicitar el conocimiento crítico y operacional de equipos de trabajo identificando: el paso a paso de lo que se hace, recursos utilizados y resultados de las actividades (Caraballo, et al., (2009). La segunda estrategia fueron las páginas amarillas (Londoño y Villareal, 2013) con las que se construyó un primer directorio de profesionales expertos de la institución con competencias específicas, cuyo objetivo fue comenzar a estimular el trabajo colaborativo al interior de la institución y el aprovechamiento de los conocimientos que disponen sus integrantes. Respecto a la tercera estrategia, esta fue denominada comunidades de práctica (Londoño y Villareal, 2013), que buscan fortalecer los procesos internos desde la dimensión ontológica, propiciando encuentros en los que se presenten -técnicas, experiencias, maneras de hacer- con el fin de intercambiar ideas y destrezas frente a acciones específicas.

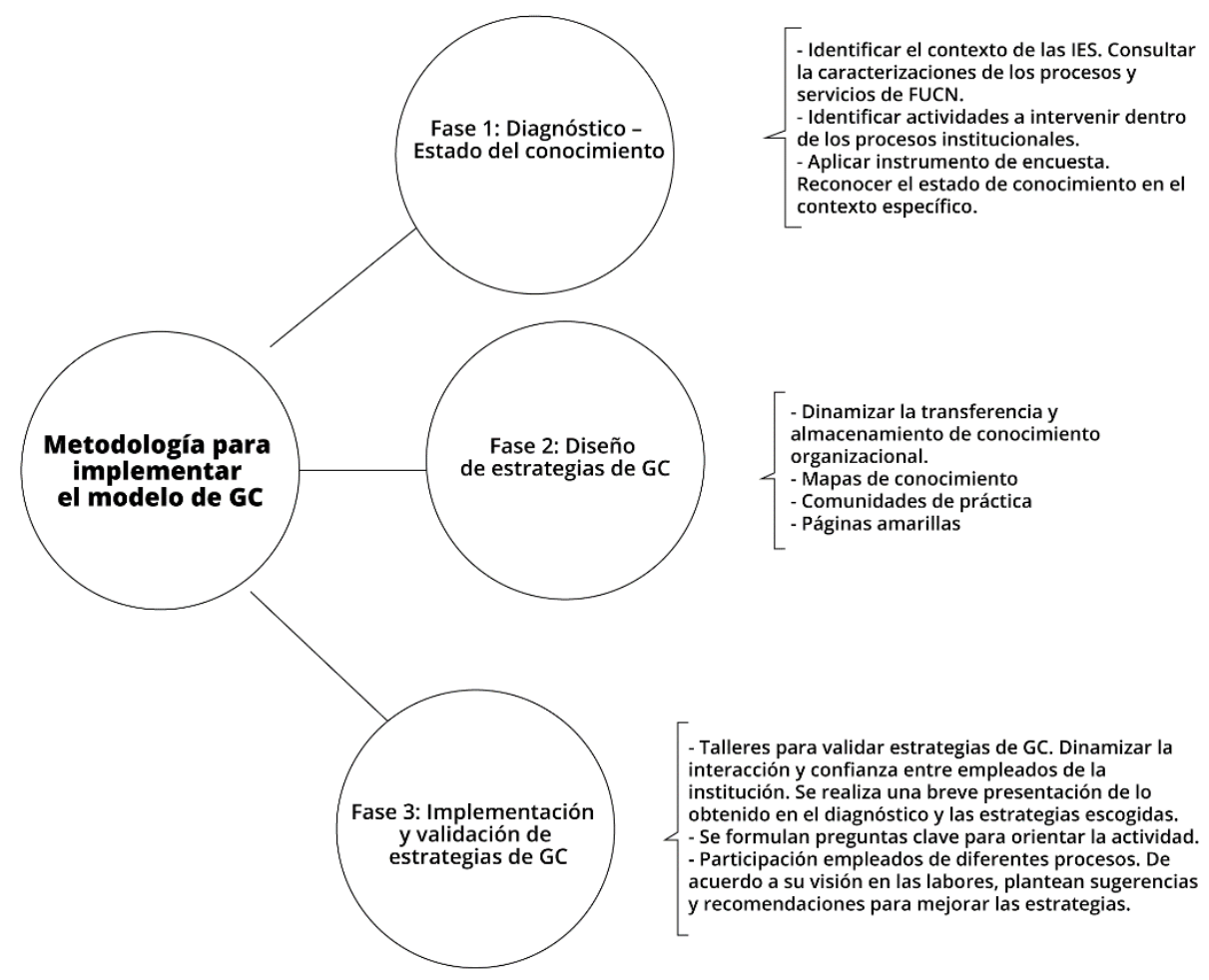

Fig. 2: Propuesta metodológica para implementar modelos de gestión del conocimiento en IES. 
En adición, para la implementación y validación de estas estrategias -acciones realizadas paralelamente-, se propuso desarrollar talleres interactivos, bajo el método del diseño de pensamiento (Mosely, et al., 2018), en aras de generar soluciones creativas a problemas y necesidades identificadas. En este espacio, participó personal de diferentes procesos administrativos, empleando la lluvia de ideas y mapas mentales como principales herramientas para evaluar las estrategias planteadas para la GC y definir su integración en sus actividades laborales. Este ejercicio, estuvo mediado por los siguientes cuestionamientos: ¿Qué creen que le hace falta al modelo? y ¿qué otras estrategias podrían implementarse acorde a los procesos a los que pertenecen? A partir de estas, se elaboró cada mapa mental intentando dejar claro aspectos como: qué uso les daría, qué se requiere para llevarlas a cabo y cómo estas mejorarían el proceso que intervienen, de esa forma se evidenció si las estrategias eran acordes a las necesidades de la FUCN. Así, se terminó la implementación del pilotaje de esta propuesta, cuyos resultados se presentan a continuación.

\section{RESULTADOS}

El diagnóstico, se diferenció de los trabajos realizados previamente por Roldán y Hernández (2011) y Sánchez (2016), en que se enfoca en conocer aspectos relacionados con los procesos y los actores en sus diferentes niveles que aportan a la: exteriorización, socialización, combinación e interiorización, así como las condiciones de las capacidades: tecnológicas, humanas y organizacionales de la institución, con el fin de encontrar aquellos procesos con mayor potencial para implementar acciones de GC y los actores con mayor vocación y receptividad a éstas.

Respecto a los procesos organizacionales y los actores relacionados con la GC valorados, se encontró que en los encuentros grupales que los dinamizan como: los grupos primarios, grupos de dirección, los comités (convivencia, educación superior, GOCl -Gestión Operativa de Calidad Institucional-) así como en las interacciones virtuales, permitían intervenciones para estimular la GC. Por otra parte, se halló que los diferentes procesos y actores cuentan con prácticas de documentación, así como con mecanismos para el almacenamiento y difusión, a pesar de que su uso se concentraba fundamentalmente en el almacenamiento. Los procesos identificados para la intervención fueron: investigaciones, planeación y calidad, y gestión humana, puesto que estos permitían de forma estratégica que el conocimiento circulara entre las personas, los diferentes niveles de la institución, así como con el contexto. En cuanto a las capacidades valoradas, se encontró lo siguiente:

Capacidad Tecnológica: La universidad cuenta con una estructura tecnológica que se dirige desde el Sistema de Gestión de Calidad, el cual provee aplicaciones web, que permiten almacenar, enriquecer y circular la información que se crea en los diferentes procesos institucionales; además cuenta con un centro de innovaciones que desarrolla aplicativos de acuerdo con las necesidades que surgen, para lo cual desarrolla y planifica acciones de mejoramiento en la calidad institucional. Sin embargo, al considerar la manera en que dichas herramientas tecnológicas aportan a la GC se encontró que su función se concentra en la exteriorización (la captura y almacenamiento de información), pero no en la socialización, combinación e interiorización, pues un 53\% percibió que ocasionalmente utiliza herramientas tecnológicas para estructurar su conocimiento, lo que se relaciona con la frecuencia en la que los actores comparten conocimiento a través de informes técnicos escritos, ya que un $29 \%$ dice que prefiere utilizar medios informales para hacerlo.

Capacidad Humana: Se constató que el conocimiento es explicitado por medio de la exteriorización y socialización, a través del diálogo que permite compartir experiencias laborales, las cuales se dan informalmente, por lo que existen dificultades para que ello redunde en procesos de codificación que permitan la combinación e interiorización de estos; en ese sentido, el $61 \%$ de los actores expresaron que suelen compartir los aprendizajes obtenidos en el desarrollo de su trabajo. Por otra parte, se encontró que el conocimiento es compartido entre personas de un mismo nivel organizacional, pero se dificulta cuando se trata de comunicarlos entre niveles, en razón a que el $69 \%$ dice que regularmente no se comparten conocimientos con compañeros de diferentes niveles jerárquicos, lo que hace que este no circule.

Capacidades Organizacionales: En esta capacidad se encontró que, si bien la FUCN promueve el aprendizaje organizacional por medio de procesos de inducción, capacitación del cargo y encuentros institucionales, no se logran promover procesos de GC que permitan explicar y codificar el conocimiento que se genera, por otra parte, se encontró que la articulación entre niveles organizacionales si bien está explicitada en la estructura organizacional, se obstaculiza la relación entre estos debido a que las lógicas cotidianas de trabajo dificultan el relacionamiento y circulación del conocimiento a nivel interorganziacional. Esto se relaciona al trabajo en conjunto que se puede realizar entre diferentes equipos de la Institución, puesto que un $51 \%$ expresa que casi no hay momentos que propicien la creación de nuevas ideas con participación de empleados de diferentes áreas.

Producto de este diagnóstico se determinó que era necesario, aprovechar las condiciones tecnológicas de la FUCN para dinamizar las interacciones entre los diferentes actores y niveles organizacionales tanto a nivel presencial como virtual, así como la exteriorización por medio de la codificación, socialización, combinación e interiorización de conocimiento. De ese mismo modo, se evidenció que, a pesar de implementar acciones 
para el manejo de información, existía un alto nivel de informalidad, que dificultaba su almacenamiento y su clasificación, así como con un mecanismo de consulta y ubicación eficiente, para que, aunque se contaba con conocimiento explícito, la dificultad para acceder a este llevaba a recurrir al conocimiento tácito.

Por otra parte, el diagnóstico reveló una débil cultura de GC, que no sólo hace parte del mundo simbólico de la organización, sino que relaciona la carencia de estrategias que la promueva, puesto que se observó que hay una resistencia frente a los cambios que pudiesen realizarse respecto a la GC, pues para ello es necesario generar lazos de confianza que fomenten la interacción y la apropiación de una cultura de GC. Por ello, se propusieron estrategias que estimulara la interacción constante entre los niveles ontológicos (individual y grupal) y epistemológicos (socialización, externalización, combinación e interiorización) en los que se desenvuelve el conocimiento al interior de la universidad, con el fin de generar dinámicas que permitan la movilización del conocimiento y de esta manera explicitar el conocimiento tácito de la universidad. Lo anterior, fomentando interacciones en los niveles individual, grupal, organizacional e interorganizacional, estimulando la socialización, exteriorización, combinación e interiorización, buscando que surta el efecto de espiral de conocimiento que proponen Nonaka y Takeuchi (1999), como se muestra en la figura 1 en el que se relaciona el movimiento esperado. Estas estrategias son: mapas de conocimiento, páginas amarillas, y comunidades de práctica.

Los mapas de conocimiento pretenden que, a partir de experiencias vividas dentro de la institución, se cree nuevo conocimiento y que este sea luego explicitado en mapas mentales como lo proponen Caraballo, et al. (2009), para lo cual periódicamente se elaboran talleres interactivos donde los participantes de los procesos ubican los conocimientos y reflexiones fruto de su quehacer, según la criticidad u operatividad de las actividades que constituyan un procedimiento. Esta estrategia se propone que los participantes exterioricen su conocimiento, y además lo modelen, de manera que este pueda ser codificado para ser socializado y transferido. Las páginas amarillas, tienen la intención de codificar la información individual de cada uno de los actores de la organización, pero al mismo tiempo clasificar estos aspectos relevantes para la FUCN, especialidades, áreas, competencias, formación académica; es decir, sea posible tener un inventario de los expertos internos, para que sean fácilmente localizados, además, esta estrategia pretende favorecer el surgimiento de redes para la creación, intercambio y transferencia de conocimiento.

Ahora bien, frente a las comunidades de práctica, estas se convierten en una estrategia que promueve tanto la combinación como la interiorización de conocimiento, puesto que además de generar un espacio en el cual es posible el tratamiento y discusión de problemáticas de la FUCN, se fomenta el desarrollo de acciones que sean ejecutadas mediante el uso de lo aprendido en espacios colaborativos. Por tanto, las estrategias diseñadas, se convierten en la primera etapa de un modelo de gestión de conocimiento, que debe incorporar otras acciones y niveles que respondan tanto a la maduración del modelo y la institución, como a orientaciones y tendencias conceptuales que aporten a mejorarlo. Los componentes que se presentan constituyen un aporte a la construcción de un modelo cuyo objetivo debe ser la gestión del conocimiento organizacional que ha sido aprendido y generado, con el fin de que este se encuentre en continuo desarrollo y actualización, propiciando la constante transferencia entre quienes hacen parte de la FUCN generando innovaciones, puesto que se promueve hacer consciente y codificar los conocimientos y las capacidades que se han generado, las cuales aportan a la interacción entre actores, al desarrollo de formas de actuación como universidad en ambientes virtuales, explícitamente a nivel de investigación, extensión y docencia, tal como se muestra en la figura 3.

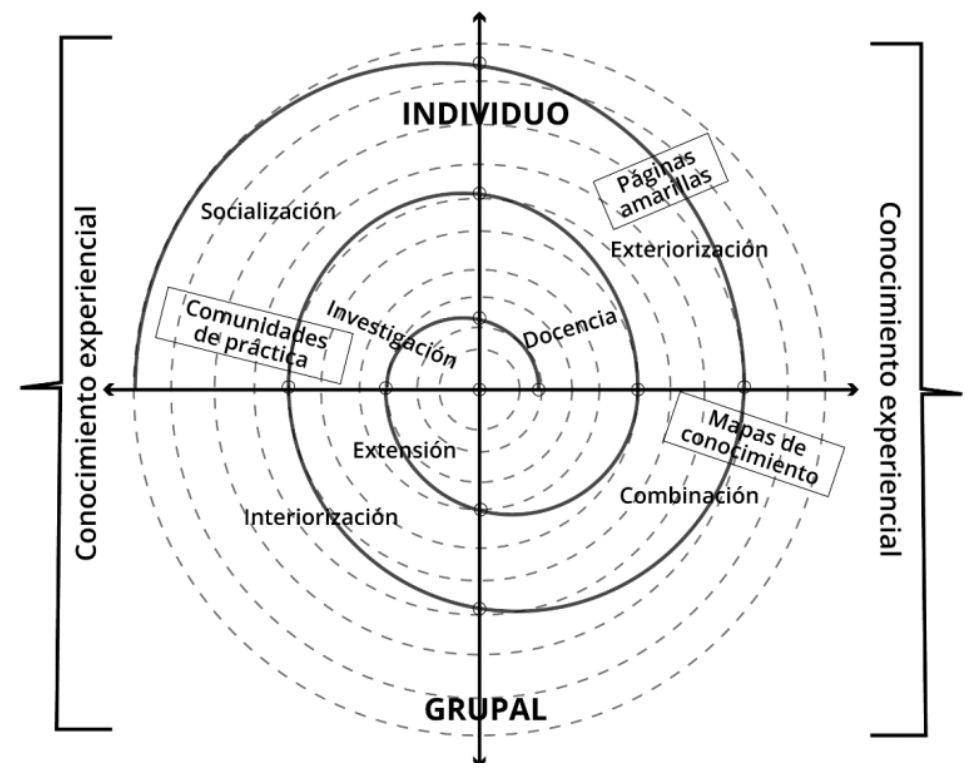

Fig. 3: Interacción entre estrategias de gestión del conocimiento y etapas de creación de conocimiento. 


\section{DISCUSIÓN}

El auge de las Tecnologías de la Información y la Comunicación -TIC-, así como el acelerado fenómeno globalizador que ha experimentado el mundo en los últimos años ha tenido un impacto significativo en las dinámicas de los países, de manera especial en los latinoamericanos. Desde la década de los noventa con las nuevas disposiciones del Consenso de Washington, los Estados de la región se vieron abocados a una serie de transformaciones políticas, sociales y económicas que ha reconfigurado la forma de asumirse a escala global, fomentando el incremento exponencial en las exportaciones (Martínez y Soto, 2012). Para ello, es crucial garantizar no sólo la productividad de las organizaciones, sino también la competitividad de los Estados, perspectiva en la cual América Latina aún tiene grandes retos.

Por lo tanto, tal como sugiere Tobón (2017), se asiste actualmente a la denominada sociedad del conocimiento, concebida como una nueva configuración social a través de la cual se espera hacer frente a grandes desafíos que se experimentan, como el cambio climático, la pobreza, el surgimiento de nuevas enfermedades, la exclusión y la violencia; lo cual demanda entre otras cosas el fortalecimiento del trabajo interdisciplinario, donde además haya una articulación clave de los diversos actores que confluyen en las sociedades.

Desde un enfoque educativo, se han propuesto dinámicas de gestión del conocimiento, entre las que se destacan el diseño del Modelo de gestión de la calidad para la educación que desarrolla Lepeley (2001), toma en cuenta el concepto de la gestión de la calidad total de Edwar Deming centralizándolo en las personas, además, se basa en tres perspectivas: Teoría Y de Douglas McGregor, Jerarquía de Necesidades Humanas y la Integración de Necesidades de las personas, con las demandas de la función y la organización. Adicionalmente, se encuentra el MGC KM como uno de los modelos más adaptados en las instituciones educativas de acuerdo con sus necesidades y contextos, el modelo tiene origen, sus cuatro pilares son: liderazgo, organización, tecnología y aprendizaje (Arceo, 2009).

Adicionalmente, si bien las universidades pueden considerarse organizaciones de conocimiento que centran su desarrollo precisamente en la producción y transferencia de este, también es claro, que su orientación hacia el desarrollo de conocimiento específicamente científico, descuida en parte aquel que se genera producto de las recurrentes experiencias de los sujetos que la componen, por lo anterior, promover estrategias que se preocupen por la captura de aquel existente tácitamente en el trabajo cotidiano de su capital humano, hasta propiciar las condiciones para que este sea explicado e incorporado a la labor cotidiana, se convierte en un primer paso hacia el desarrollo de un modelo de gestión de conocimiento centrado en el aprendizaje organizacional.

De este modo, estrategias como los mapas de conocimiento, propician retomar del ambiente particular de trabajo, los datos que son explicados por los actores, como conceptos que pueden ser de igual forma combinados con los de otros, de modo que la estrategia precisamente apunta a una subsanar esa dificultad diagnosticada por González, et al., (2004) en las IES, respecto a la incapacidad de gestionar su aprendizaje, proceso en el que la universidad explicite lo aprendido y lo compartido, para que actúe como impulsor del espiral propuesto por Nonaka y Takeuchi (1999). Y así mismo, se tome el tiempo para reflexionar sobre los conocimientos de tal manera que tenga la capacidad para hacer que estos puedan ser incorporados en contexto en las diferentes áreas de la universidad.

Así, al explorar la literatura es posible evidenciar algunas aproximaciones respecto a la utilidad de los mapas de conocimiento, como es el caso del estudio de García-Álvarez (2013), quien los cataloga como un sistema de información para la gestión del conocimiento, convirtiéndose en una herramienta útil para la adquisición de saberes por parte de los integrantes de una organización, en lo cual también coinciden Guitián y Dante (2011), quienes además resaltan la relevancia de estos mapas para la visualización gráfica del conocimiento generado y empleado por las personas, develando también los mecanismos para su almacenamiento y transferencia a nivel grupal e individual.

De otro lado, Ramírez (2013) resalta la importancia de estos mapas en el contexto de las IES dado que posibilitan entre otras cosas, contar con un registro del conocimiento existente, localizarlo y rastrear la experiencia del talento humano del que disponen las organizaciones, además de poder visualizar las formas en que dichos conocimientos existentes interactúan. Para ello, la autora presenta el caso específico implementado en la ciudad de Bucaramanga (Colombia) en la seccional de la Universidad Pontificia Bolivariana, a través del diseño de una estrategia para la construcción de un mapa de conocimiento para la Dirección de Investigaciones de dicha institución.

Por su parte, la ya referida dinámica de espiral se estimula también al promover la interacción entre los diferentes actores universitarios, para ello es importante el papel que juegan las páginas amarillas, puesto 
que estas pretenden mantener y generar interacciones, en las que se lleva la socialización a niveles que superen los grupos primarios $u$ otras actividades colectivas como los mismos mapas de conocimiento, para que esta no se dé solo en momentos específicos en el tiempo, sino que también se estimule al tener la posibilidad de interactuar con aquellos expertos de la institución en las necesidades que se presentan. Consecuentemente, como lo proponen Yusof et al. (2012), se promueve la adquisición de habilidades en los diferentes niveles de la organización y los actores que la conforman, haciéndoles partícipes del desarrollo de conocimiento en la institución universitaria.

Frente a esta estrategia, es conveniente señalar el estudio realizado por De Freitas y Yáber (2015) para el contexto de las Instituciones de Educación Superior, a través del cual identifican algunas herramientas cuyo propósito es similar al que se esboza en este manuscrito frente a las páginas amarillas, quienes las catalogan como una herramienta de tipo colaborativo, es decir, aquellas a través de las cuales las organizaciones esperan externalizar conocimientos específicos; junto con otras estrategias como las comunidades virtuales, los chat, conocimientos relacionados y grupos colaborativos, donde pueden inscribirse por su intencionalidad las comunidades de práctica.

Por su parte, Gelabert y Martínez (2012) presentan en su estudio a partir de una revisión de literatura, las forma como la gestión de recursos humanos contribuyen a la gestión del conocimiento, develando así que en la dimensión de aprendizaje -abordada por esta área en las diferentes organizaciones-, existe un beneficio potencial de las comunidades de práctica como espacios a través de los cuales se convierte el conocimiento tácito en explícito, donde el aprendizaje se articula en la creación de rutinas para mejorar el desempeño de las compañías. Además, las comunidades de práctica como escenarios de trabajo colectivo que permiten mantenerse en el tiempo, cumplen una condición fundamental para el proceso de gestión del conocimiento universitario, puesto que funcionan tanto como escenario de combinación como de interiorización; estas articulan esencialmente las dos estrategias que conforman esta propuesta, en tanto interrelacionan el conocimiento explicitado con un proceso de reflexión, el cual permite que los grupos en este caso, articulen y desarrollen nuevos conceptos que pueden ser incorporados como parte de los aprendizajes.

Por ende, las estrategias se vinculan para dinamizar el conocimiento institucional, y así, promover el desarrollo de acciones que comiencen a incorporar en la organización ideas y hábitos que funcionen como promotores de la gestión, de tal forma que con el continuo movimiento las dimensiones que se pretenden impactar con ello, permitan por un lado aportar al desarrollo de una cultura que entienda el valor del conocimiento y lo gestione, y por otra, aprovechar este conocimiento para generar condiciones que permitan el aprovechamiento de este para ser usados estratégicamente (Asma y Abdellatif, 2016), en las diferentes actividades que la universidad desarrolla para cumplir su misión en extensión, investigación y docencia.

Consecuentemente, vale destacar que el modelo planteado crea retos para la comunidad científica y académica, al constituirse en un llamado para trascender las acciones de generación de nuevo conocimiento, formación de talento humano y desarrollo tecnológico; comprendiendo que la esencia de las IES, si bien es la prestación de un bien público meritorio, las universidades y demás establecimientos de formación deben comenzar a administrarse bajo una óptica gerencial, que permita generar un mayor impacto en sus funciones sustantivas de docencia, extensión e investigación, por lo cual gestionar el conocimiento es una actividad clave para poder migrar hacia las nuevas tendencias que pone esta llamada sociedad del conocimiento. Esto es congruente con los planteamientos de Di Nauta, et al. (2018) quienes aseveran los constantes cambios que ha implicado la sociedad del conocimiento, y cómo las Instituciones de Educación Superior han tomado consciencia respecto al valor que adquiere el conocimiento para la generación de ingresos, su diferenciación y productividad en el mercado.

\section{CONCLUSIONES}

1. La formulación propuesta en este artículo ha permitido entender la utilidad de realizar un diagnóstico previo con el fin de contextualizar el panorama de intervención y generar mapas de conocimiento orientados a concientizar de los procedimientos institucionales como mecanismo para explicitar y apropiar los conocimientos relevantes.

2. Se propone la comprensión del contexto y las lógicas de la institución donde se ejecutará y validará el modelo, pues esto define las dinámicas y particularidades que determinan la circulación, almacenamiento, apropiación y transferencia del conocimiento, para el reconocimiento de condiciones organizacionales, tecnológica y humana.

3. Los mapas de conocimiento posibilitan una mayor consciencia frente a los procedimientos inmersos en las dinámicas institucionales, ya que este proceso permite fortalecer de forma más estructural la construcción y suministro de información al modelo de gestión de conocimiento, haciéndolo más robusto y aplicable a medida que involucra procesos más diversos. 
4. Uno de los recursos más efectivos en la formulación del modelo son las páginas amarillas, posicionándose como una forma de almacenar información de expertos y de empleados de las Instituciones de Educación Superior, esto con el fin de retenerla y ponerla a disposición de toda la comunidad docentes y administrativa. Lo anterior, se complementa con las comunidades de práctica como estrategia para fomentar la transferencia de conocimiento a través de encuentros programados para la difusión de nuevos conocimientos al interior de la institución.

5. El propósito fundamental de un modelo de gestión del conocimiento en IES se debe orientar al fortalecimiento de la cultura alrededor de estrategias de preservación de conocimiento, lo cual se articula con la creación, codificación, apropiación y la reutilización de los conocimientos ya existentes y que, si se acompaña con prácticas estructuradas, permite la consolidación de experiencias que fomenten la apropiación y transferencia de conocimiento.

\section{REFERENCIAS}

Arceo, A., Gestión del conocimiento en educación y transformación de la escuela. Notas para un campo en construcción, ISSN: 2007-7033. Sinéctica, revista electrónica de educación, (32), 1-21. (2009).

Arévalo-Avecillas, D., S. Ñájera-Acuña y Piñero, E. A., La Influencia de la Implementación de las Tecnologías de Información en la Productividad de Empresas de Servicios. doi: http://dx.doi.org/10.4067/S0718-07642018000600199. Información Tecnológica, 29(6) (2018).

Arias, J. E y Aristizábal, C.A., Transferencia de conocimiento orientada a la innovación social en la relación cienciatecnología y sociedad; ISSN: 1657 - 6276, Pensamiento y Gestión, (31), 137-166 (2011).

Asma, $\mathrm{K}$ y M. Abdellatif., A New Model for the Impact of Knowledge Management on University Performance. Part 1: Theoretical Developmen, doi: 10.1142/S0219649216500416, Journal of Information \& Knowledge Management, 15 (4) (2016).

Caraballo, Y., Mesa, D. y Herrera, J. A., Herramientas de gestión del conocimiento: convergencias hacia un aprendizaje organizacional; ISSN: 0034-7485, Revista Cubana de Ciencia Agrícola, 43 (1), 1-13 (2009).

Chen, F. y Burstein, F., A dynamic model of knowledge management for higher education development. 7th International Conference on Information Technology Based Higher Education and Training, 173-180, doi: 10.1109/ITHET.2006.339762 (2006).

De Freitas V., y Oltra, G. Y., Una propuesta de arquitectura para los Sistemas Informáticos de Gestión del Conocimiento en Instituciones de Educación Superior, ISSN: 0798 1015, Revista ESPACIOS, 36(10) (2015).

De Molero, N., Contreras, G., y Casanova, R., Knowledge management as a tool for the productivity of research in the university sector; ISSN: 1856-9331, REDHECS, (21), 147-165 (2017).

De Moortel K. y Crispeels, T., International university-university technology transfer: Strategic management framework, doi: 10.1016/j.techfore.2018.05.002, Technological Forecasting and Social Change, 135, 145-155 (2018).

Di Nauta, P., B. Merola., y otros dos autores, Reflections on the Role of University to Face the Challenges of Knowledge Society for the Local Economic Development, doi: https://doi.org/10.1007/s1313, Journal of the Knowledge Economy, 9 (iss. 1), 180 - 198 (2018).

Fundación Universitaria Católica del Norte, Plan de desarrollo 2016-2020 (2016).

García-Álvarez, M. T., El rol de las tecnologías de la información y comunicación en la gestión del conocimiento: un desafío estratégico en el nuevo contexto empresarial; ISSN: 1315-9518, Revista de Ciencias Sociales (Ve), 19(2), 322333. (2013).

Gelabert, C. M., y Martinez, A. A., Contribución de la gestión de recursos humanos a la gestión del conocimiento, ISSN: 0123-5923, Estudios gerenciales, 28(123), 133-148 (2012).

González, A.L., Castro, J. P. y Roncallo, M., Diagnóstico de la gestión del conocimiento en una empresa grande de Barranquilla (Colombia), ISSN: 0122 - 3461, Ingeniería y Desarrollo, (16), 70-103. Universidad del Norte (2004).

Guitián, M. V. G., y Dante, G. P., Mirada contextual a los nexos entre las auditorías de información y las auditorías de conocimiento, ISSN: 0864-4659, Ciencias de la Información, 42(1), 31-37. Instituto de Información Científica y Tecnológica (2011).

Hosseini, S. S., Nikkhah Tekmedash, Y. y otros dos autores , Y, The Impact of Knowledge Management Strategy on Service Innovation Performance in Private and Public Hospitals, doi: https://doi.org/10.22059/ijms.2018.249784.672966; Iranian Journal of Management Studies, 12(1), 1-24 (2019).

Lepeley, M. T., Gestión y calidad en educación: un modelo de evaluación (p. 4). McGraw-Hill Interamericana, México (2001).

Londoño, M. y Villareal, R., Aproximación a un modelo de gestión de conocimiento. El caso de la Facultad de Comunicaciones de la Universidad de Antioquia, Tesis de maestría. Universidad de Antioquia, Medellín, Colombia (2013). 
Martínez, R. y Soto., E., El Consenso de Washington: la instauración de las políticas neoliberales en América Latina; goo.gl/p8cgHB, ISSN: 0188 - 7742, Política y Cultura, (37), 35 - 64 (2012).

Marulanda, C. E., Valencia, F. J. y Marín, P. F., Principales Obstáculos para la Transferencia de Conocimiento en los Centros e Institutos de Investigación del Triángulo del Café en Colombia, doi: http://dx.doi.org/10.4067/S071807642019000300039; Información Tecnológica, 30(3) (2019).

Michelino, F., E. Lamberti y otros dos autores, Open models for innovation: an accounting-based perspective, doi: 10.1504/IJTM.2015.068778; International Journal of Technology Management, 68(1-2), 99-121 (2015).

Moffett, S., McAdam, R., y Parkinson, S., Developing a model for technology and cultural factors in knowledge management: a factor analysis, https://doi.org/10.1002/kpm.152, Knowledge and Process Management 9(4), $237-255$. (2002).

Mosely, G., N. Wright y C. Wrigley., Facilitating design thinking: A comparison of design expertise, doi: 10.1016/j.tsc.2018.02.004; Thinking Skills and Creativity, 27, 177-189 (2018).

Natek, S y M. Zwilling, Student data mining solution-knowledge management system related to higher education institutions, doi: 10.1016/j.eswa.2014.04.024, Expert Systems with Applications, 41 (14), 6400-6407 (2014).

Nonaka, I y H. Takeuchi, La organización creadora de conocimiento. Cómo las compañías japonesas crean la dinámica de la innovación. Oxford University Press, México DF (1999).

Ramírez, G., Construcción de mapas de conocimiento en las Universidades, ISSN: 0120-1115, Revista Universidad Pontificia Bolivariana 53(153), 65-78 (2013).

Roldán, N. D. y Hernández, J. E., Hacia un modelo de gestión del conocimiento en la Fundación Universitaria Católica del Norte. Medellín, Antioquia, Colombia (2011).

Rodríguez E., Cohen, W., y otros tres autores. La gestión del conocimiento y la calidad de la docencia de postgrado en las universidades: un estudio exploratorio, doi: 10.15446/innovar.v24n52.42506, Innovar, 24 (52), 59-66 (2014).

Saad, N.H.M., Kasimin, H., y otros dos autores, Soft systems methodology a conceptual model of knowledge management systems initiatives in malaysian public universities, doi: 10.1007/978-3-642-54105-6_25, KMIS 2012 - Proceedings of the International Conference on Knowledge Management and Information Sharing, 60-69 (2012).

Sánchez, L. F., Proyección del MISE como modelo de sistematización de experiencias en la Fundación Universitaria Católica del Norte; Tesis de pregrado. Universidad de Antioquia, Medellín, Colombia (2016).

Sein, M.L., Fidalgo, A, y otros dos autores, The learning improvement of engineering students using peer-created complementary resources, ISSN: 0949 - 149X, International Journal of Engineering Education, 33 (2), $927-937$ (2017).

Simanjuntak M., S.A, Manalu y R.D Tobing., Towards a institude of technology: A knowledge management system challenges in the perspective of academic and student administration bureau, doi: 10.11113/jt.v78.8931, Jurnal Teknologi, $78(6-3), 71-76(2016)$.

Tobón, S. Ejes esenciales de la sociedad del conocimiento y la socioformación. Mount Dora: Kresearch, doi: 10.24944/isbn.978-1-945721-18-2 (2017).

UNESCO, Conferencia mundial sobre Educación Superior - 2009: La nueva dinámica de la educación superior y la investigación para el cambio social y el desarrollo (2009).

Villafuerte-Godínez, R. y Leiva J.C., Cómo surge y se vincula el conocimiento relacionado con el desempeño en las Pymes: un análisis cualitativo, doi: 10.22430/24223182.123, Revista CEA, 1(2), 37-48 (2015).

Yusof, A., Ahmad, N., y otros dos autores, Quality and effectiveness of knowledge management transfer using of mentormentee program and on job training in work place, doi: 10.1109/ICIMTR.2012.6236359, 2012 Int. Conf. on Innovation, Management and Technology Research (ICIMTR2012), Malacca, Malaysia 21 - 22 de mayo (2012). 